\title{
Symposium über formelle und informelle Soziale Sicherheit
}

\author{
Von Ingo Winkelmann
}

Vom 2. bis 6. Juni 1986 fand in den Einrichtungen der am diesmal leider verregneten Starnberger See gelegenen Akademie für politische Bildung in Tutzing ein internationales Symposium über Formen "formeller" und "informeller" Sozialer Sicherheit unter besonderer Berücksichtigung der Staaten der Dritten Welt statt. Unter Federführung des gastgebenden Münchner Max-Planck-Instituts für Ausländisches und Internationales Sozialrecht (MPI/AIS) und der International Commission on Folk Law and Legal Pluralism (ICFLLP), die zusammen die Tagung veranstalteten, hatte sich ein namhafter und internationaler Teilnehmerkreis eingefunden.

Weniger diese Seite der Tagung und deren bewußt über innerstaatliche Bezüge hinausgehende thematische Ausrichtung verdienten jedoch besondere Aufmerksamkeit, als die Tatsache eines in dieser Form wohl nicht häufig anzutreffenden, teils sozialrechtlich, teils sozialpolitisch gelagerten Experiments direkten interdisziplinären Austauschs zwischen Rechtswissenschaftlern, Rechtssoziologen, Okonomen auf der "einen" und Entwicklungsexperten, Anthropologen und Ethnologen auf der "anderen" Seite. Es hatte wohl gerade der Initiative zweier verhältnismäßig junger Institutionen wie jener der eingangs genannten Veranstalter bedurft - während die Gründung der ICFLLP-Gruppe durch die International Union of Anthropological and Ethnological Sciences (IUAEL) auf das Jahr 1978 zurückgeht, konnte das MPI/AIS in diesem Jahr immerhin schon sein zehnjähriges Bestehen feiern -, um auf solch ungewohnten und hinsichtlich der einzukalkulierenden Verständnisschwierigkeiten nicht ganz risikolosen Wegen das Thema Soziale Sicherheit in der Dritten Welt einer sehr großzügig umrissenen Untersuchung zu unterziehen. Der Reiz, der mit dieser Symposiumskonzeption verknüpft war, mag mit diesen wenigen Vorbemerkungen genügend skizziert sein, ebenso die Reibungsflächen angedeutet, die ein Aufeinanderprallen derart unterschiedlicher Anliegen, Methoden und Aussagegewichtungen von Beginn an erwarten ließ. Doch dazu später. Eingeteilt war das - englischsprachige - Tagungsprogramm in vier unterschiedlich umfangreiche Themenblöcke:

\section{Teil A: On the development of formal social security in developing countries}

Im Hauptbeitrag zu diesem Veranstaltungsteil befaßte sich Fuchs ausführlich mit der Entwicklung und den Zukunftsaussichten formeller Systeme Sozialer Sicherheit in Dritteweltstaaten. Seine Aussagen trafen auf ein Auditorium, das noch unter dem Gesamteindruck stand, den die beiden vorhergehenden Eröffnungsreferate hinterlassen hatten. 
Von Beginn an waren nämlich die gegensätzlichen Sichtweisen und Blickwinkel plastisch geworden, unter denen die beteiligten Seiten den Tagungsgegenstand in Angriff zu nehmen gedachten: Einerseits ein entschiedenes Plädoyer zur Stärkung der Komplementärwirkung sowohl der (regelmäßigen) informellen, wie auch der (lediglich fragmentarischen) formellen Sicherungssysteme, deren Definier- und Abgrenzbarkeit vorausgesetzt wurden (Zacher), andererseits eine betont skeptische Akzentuierung der Tatsache, daß auf local level in Afrika und Lateinamerika formelle und informelle Systeme und deren Merkmale eben solcher Unterscheidbarkeit und Kategorienbildung nur schwerlich zugänglich seien (F. von Benda-Beckmann), es mithin eines gänzlich anderen Lösungsansatzes bedürfe. Unübersehbar indizierten beide Auffassungen jeweils unterschiedliche Bedeutungszumessungen an terminologische Fragen.

\section{Teil B: Non-state regulated social security and change}

Auf der Grundlage des anthropologischen Konzepts der inclusionary transition führte Casiño die Zuhörer von den ehemaligen "Flußmündungskönigtürmern" Südostasiens und deren Eigenheiten über zurückliegende Zeiten von Sklaverei und Sklaventum bis hin zur Forderung nach "moralischer Integrität" des staatlichen Gesetzgebers, s. A. nach eines der Hauptprobleme der südostasiatischen Staaten von heute.

Schott befaßte sich dann mit der Krise "traditioneller Sicherungssysteme" in Westafrika; anhand der Schilderung zahlreicher Fallstudien in Burkina Faso (ehem. Obervolta) und Ghana wurde deutlich, in welchem Maße die Solidarität überkommener Dorfverbände (compounds) nicht nur schon seit jeher, sondern besonders im Zuge der gegenwärtigen ökonomischen Krisen rissig geworden ist. Hieran schlossen sich erste Ergebnisse eines Feldaufenthalts in Macabaclay (Phillipinen) an, von dem Hirtz berichtete.

Die einsetzende Zerrüttung der moralischen Verpflichtung von Familiennachkommen in Korea, ihre Eltern im Alter zu unterhalten (hyo-song) - verschärft durch die faktische Inexistenz dortiger staatlicher Alterssicherung -, war Gegenstand eines Berichts von Kim.

Nachdem Griffith ihren sorgfältig analysierenden Bericht über (vier) verschiedene Arten sozialer "Unterstützung" innerhalb ausgewählter Familienhaushalte des botswanesischen Stammes der bakwena vorgetragen hatte, stellte Wanitzek dar, in welcher Weise hergebrachte familiäre Sicherungsformen in Tansania zwar mit der Zeit verloren gegangen sind, doch heute umgekehrt in Gestalt moderner Gesetzgebungsbemühungen wiederaufgenommen werden, Beispiel: Entschädigungsklagen lediger Mütter (misango). "Informelle Soziale Sicherheit unter molukkischen Immigranten in den Niederlanden" hieß der Titel des Beitrages von Strijbosch; von einem Dorf auf den Molukken (dem ambonesischen Hila) und überkommenen (islamischen) Sicherungsinstituten wie etwa der "Almosensteuer" (zakat) berichtete im Anschluß daran F. von Benda-Beckmann (auf die spezifische Rolle ambonesischer Kleinhändler ging K. von Benda-Beckmann später im folgenden Teil C ein). 


\section{Teil C: Co-existence and pluralism of social security systems}

Der detaillierten und in ihrer Nachdenklichkeit beeindruckenden Auseinandersetzung Woodmans mit dem of fenbaren Niedergang traditioneller Formen Sozialer Sicherheit in den englischsprachigen Ländern Afrikas folgten gleich zwei Kontributionen, die sich erneut mit Tansania beschäftigten: ein schwerpunktmäßig entwicklungsgeschichtlich angelegter Beitrag von Bossert sowie eine Untersuchung dortiger urbaner Siedlungszentren (urban centers), die Bakari mit mehreren Fallstudien belegte. Einer der Tagungshöhepunkte war das sich anschließende Referat von Kludze, der (am Beispiel Ghanas) in unnachahmlicher Weise die Unzulänglichkeit bestehender formeller Sicherungssysteme in Afrika anschaulich zu machen wußte. Wieder in ganz andere geographische Breiten führten die Darlegungen von Boaventura de Sousa Santos. Ihm gelang es, die soziale Absicherung und generelle Bedeutung der portugiesischen ländlichen Bevölkerung für die Gesamtgesellschaft unter besonderer Würdigung politischer Faktoren nachzuzeichnen. Auf einem stark wirtschaftstheoretisch konzipierten Ansatz beruhte die Feldstudie von Freiberg/Jung über die Änderung von Erwerbsstrukturen in kolumbianischen ländlichen Familienverbänden im Raum Boyacá. Schon vorher war es Chang beschieden gewesen, am Beispiel Taiwans die Gültigkeit terminologischer Unterscheidungen zwischen "formellen" und "informellen" Sozialsystemen für Länder der Dritten Welt und sog. "Schwellenländer" einer kritischen Würdigung zu unterziehen.

\section{Teil D: Procedures, working of social security institutions, conflicts and adaptions}

Als erste beschrieb Pijuan am Beispiel der Phillipinen die Möglichkeit der Durchsetzung sozialer Rechte vor Gericht. Der nachfolgende Bericht von Slaats/Portier hatte Verfahrensweisen und Probleme der Bodenvergabe im Stamm der karo auf Sumatra zum Inhalt. Ähnlich verschieden waren auch die Themen zweier weiterer Beiträge: die Handhabung migrationsbedingter sozialer Probleme in Afrika durch internationale Abkommen (Kaufmann) und die Spannungslage zwischen unterer (Sozial)Bürokratieebene und Leistungsanspruchsinhabern in den Niederlanden (de Koning). Schon zum Schlußtag gehörten zwei Länderbetrachtungen, in deren Rahmen Azer auf die zwiespältige Rolle fundamentalistischer Religionsgruppen und die Notwendigkeit der Begehung neuer, sozialwirksamer(er) Wege für Ägypten hinwies und Conn sich bemühte, für den Fall Alaska das Konzept der retribalization in einem sozialen und gesamtgesellschaftlichen Zusammenhang fruchtbar zu machen.

Nicht zu beneiden war am Ende Bryde um die Aufgabe eines zusammenfassenden Schlußreferats. Um so stärkere Bewunderung verdiente die gleichermaßen präzise wie ausgewogene Art, mit welcher er sich dieses Auftrags entledigte. Hiervon zeugte nicht zuletzt die ebenso geschickt wie berechtigt in den Mittelpunkt seiner Uberlegungen plazierte Feststellung, daß die zutagegetretene Variationsbreite der vorgestellten Beiträge sicherlich mehr Fragen aufgeworfen denn konkrete Lösungen angeboten hätte. 
Geschickt, insoweit damit entschärft wurde, was im Laufe des Symposiums an methodologischen Differenzen, unterschiedlichen Zielvorgaben und Verständnisproblemen zutagegetreten war. Insbesondere ausfüllungsbedürftige Begriffe wie "(Sub)Normalität", "Subsistenzwirtschaft", "Semikapitalismus", "Semiperipherieländer" u. a. m. hatten im ersten Moment eher Uneinigkeit denn den - oft vermißten - Brückenschlag von (konkreten) Fallstudien zu gemeinsamen, abstrahierungszugänglichen Lösungsansätzen bewirkt.

Berechtigt, da zugleich aber auch eine spürbare Problemsensibilisierung für die Dringlichkeit gelungen war, mit der eine Großzahl teilweise schon bereits geklärt beglaubter Fragen weiterhin ihrer Lösung harrten: Woran etwa würde ein einheitlich verwendbarer Begriff von "Sozialer Sicherheit" sich messen lassen müssen? In welchem Maß können Grenzziehungen zwischen "formeller" und "informeller" Sicherung in der Dritten Welt überhaupt gelingen? Wären andere Begriffe - wie etwa jener einer (auszudifferenzierenden) "social protection" - nicht womöglich adäquater? Besteht dort, wo Sozialpolitik eine Stärkung des (nichtstaatlichen) sozialen "Rückgrats" Familie vernachlässigt, nicht die Gefahr einer Uberlastung der schon als solchen vielfach um innere Stabilität ringenden Staatengebilde der Dritten Welt? Welches wären die hieraus zwangsläufig resultierenden, miteinzubeziehenden politischen Faktoren? Uneingeschränkten Beifall verdiente schließlich auch Brydes Hinweis auf die nur begrenzte Ubertragbarkeit westlich konzipierter Gestaltungen von "formellem" und "informellem" Sektor in die Staaten der Dritten Welt; von hier zu der naheliegenden Frage nach den generellen, nicht zuletzt kulturellen Grenzen der Adaptionsfähigkeit von an okzidentalen Maßstäben orientierten Sozialsystemen in der Dritten Welt war es dann nur noch ein kurzer Schritt.

Fazit: Trotz vieler Fragezeichen und mancher Verwirrung muß das Tagungsexperiment als geglückt und als ein Ansporn zu weiterem Beschreiten des eingeschlagenen Weges angesehen werden: zu drängend sind die einer Systematisierung und Kanalisierung harrenden sozialen Probleme der Dritten Welt, zu sehr bedarf die Politik der Anregungen praxisnaher Forschung, wie sie sich auf dieser Tagung geballt präsentierte.

Daneben wurden einmal Nichtjuristen die Bedeutung von Begriffserklärungen und -abgrenzungen für Verfahrenserleichterung und Lösungsfindung vor Augen geführt, den Juristen widerum - und hier eben gerade auch den Sozialrechtlern unter ihnen - bewußt gemacht, in welchem Maße Vorgaben und Prämissen der vorherigen Beteiligung aller benachbarten Disziplinen bedürfen, um einer derart komplexen Thematik wie der von Sozialer Sicherheit in der Dritten Welt gerecht werden zu können. Nicht unziemlich erscheint an dieser Stelle der Hinweis auf den auch weiterhin andauernden Lernbedarf an Verständnisfähigkeit und -bereitschaft bei allen Beteiligten.

Somit standen die fünf Tutzinger Tage für eine Aufforderung an Veranstalter und Teilnehmer zu Kontinuität, weiterer empirischer Materialsammlung und sich daran anschließender Materialauswertung. Der geplanten Veröffentlichung der Symposiumsbeiträge und -diskussionen darf man mit großer Spannung entgegensehen. 